\title{
Consecuencias neurocognitivas de los trastornos respiratorios del sueño
}

\author{
Dr. Pablo E. Brockmann
}

1 Profesor Asistente, División de Pediatría, Escuela de Medicina. Pontificia Universidad Católica de Chile.

\section{NEUROCOGNITIVE CONSEQUENCES OF SLEEP BREATHING DISORDER}

Sleep disordered breathing affect almost 10\% of the pediatric population. There is consistent evidence that supports an association between sleep disordered breathing and neurocognitive consequences like hyperactivity, poor school performance, aggressiveness, and somnolence. There is also a dose-effect and molecular relationship between this variables that may lead to irreversible damage of the developing nervous system. Not only obstructive sleep apnea may have neurocognitive consequences, primary snorers may also show this adverse development. Early detection and treatment may stop the progression of long-term neurocognitive consequences. Key words: Sleep, apnea, snoring, neurocognitive, school performance.

\section{RESUMEN}

Los trastornos respiratorios del sueño (TRS) afectan aproximadamente al 10\% de la población pediátrica. Existe evidencia considerable que sustenta una asociación causal entre los TRS y efectos neurocognitivos en niños como hiperactividad, mal rendimiento escolar, agresividad y somnolencia. Existe asociación de tipo dosis-efecto y molecular que demostraría un posible daño irreparable a zonas susceptibles del cerebro en desarrollo; no tan sólo el síndrome de apnea obstructiva del sueño, sino también los roncadores primarios pueden tener consecuencias neurocognitivos. Una detección precoz y tratamiento oportuno de los TRS pudiera detener la progresión de las consecuencias neurocognitivas a largo plazo.

Palabras clave: Sueño, apneas, ronquido, neurocognitivos, rendimiento escolar.

\section{INTRODUCCIÓN Y DEFINICIONES}

"Joe! Joe!" dijo el Sr. Wardle. "Despierten a ese niño de mejillas rojas! Nuevamente se durmió. Pínchenle una pierna, sólo así despierta. Muchas gracias. Trae el almuerzo Joe, por favor... " El gordito, que lentamente despertó, procedió a desempacar el almuerzo, muy lentamente, hasta que de nuevo se durmió y comenzó a roncar... ".

Charles Dickens, The posthumous Papers of the Pick-Wick Club, 1837

Los trastornos respiratorios del sueño (TRS) se presentan en el $10 \%$ de la población pediátrica( ${ }^{(1)}$, presentando un espectro variable de enfermedad. En el extremo de menor severidad se ubican los llamados "roncadores primarios"; los cuales representan la mayor parte de los TRS y se caracterizan por presentar ronquido frecuente por más de 3 veces a la semana; sin embargo, no presentan apneas ni desaturaciones visibles en la polisomnografía (PSG). El grupo intermedio de severidad corresponde al "síndrome de aumento de la resistencia de la vía aérea superior"; el cual además de presentar

Correspondencia: Dr. Pablo E. Brockmann

División de Pediatría. Lira 85, 5to piso, Santiago, Chile. Telelefono: 56(2) 3543767.

E mail: pbrockmann@med.puc.cl

ISSN 07| 8-332| Derechos reservados. ronquido frecuente, presenta la aparición de microdespertares frecuentes y signos directos o indirectos de un aumento de la resistencia de la vía aérea superior en la PSG(2). El I-2\% de la población pediátrica general presenta el "síndrome de apnea obstructivas del sueño (SAOS)", el cual se define actualmente como la presencia de un índice de apnea-hipopnea por hora $>1,0$ en la PSG(3). Otros puntos de corte no se recomiendan actualmente para esta definición en niños que se basa en una PSG. La figura I resumen los hallazgos característicos de cada uno de los componentes de los TRS.

Las consecuencias de los TRS han sido documentadas desde hace décadas, en especial en adultos. En niños, se han descrito complicaciones cardiovasculares, como hipertensión arterial y pulmonar( ${ }^{(4)}$, síndrome metabólico(5), además de una serie de consecuencias neurocognitivas. Las consecuencias neurocognitivas de los TRS han sido evidenciadas en múltiples estudios publicados en los últimos 20 años. La evidencia actualmente disponible que sustenta la asociación de los TRS con consecuencias neurocognitivas será detallada a continuación.

\section{CONSECUENCIAS NEUROCOGNITIVAS}

El reconocimiento que un mal dormir puede traer consecuencias en el desempeño durante la vigilia se ha reconocido desde hace más de un siglo ${ }^{(6)}$, estudios en adultos en los cua- 


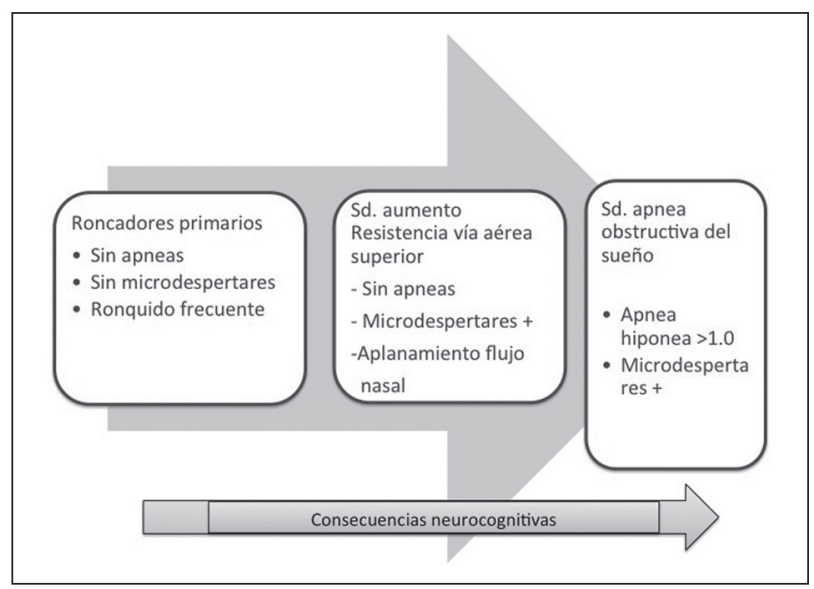

Figura I. Hallazgos característicos de la TRS en niños.

les se realizaba una deprivación de sueño demostraban que el desempeño en la realización de un sinnúmero de tareas de memoria, visuales y de trabajo disminuía en comparación con aquellos que dormían de forma normal(7). Como estos hallazgos en población adulta pudieran afectar a niños había sido un tema de hipótesis y debate durante muchos años hasta que nueva evidencia demostró un efecto importante de los TRS sobre el desempeño neurocognitivo en niños y adolescentes.

Uno de los primeros estudios que reposicionó la hipótesis de las consecuencias neurocognitivas producidas por los TRS fue publicado en 1998 por Gozal( ${ }^{(8)}$. En este estudio se demostró en 270 escolares una asociación de mal rendimiento escolar y ronquido habitual. Aquellos niños que presentaban las peores notas escolares (menor al percentil 10 de rendimiento), eran los que más TRS presentaban. En los sujetos que se sometieron a una adenotonsilectomía, se observó una mejoría significativa de las notas en comparación a aquellos sujetos en los cuales no se intervino(8). Estudios posteriores han ratificado esta asociación, entre ellos destaca el estudio realizado en la ciudad alemana de Hannover el 200 I, el cual demostró en I.| 44 escolares de 3er año básico, un efecto dosis-respuesta, es decir: mientras más frecuente roncaban los niños, presentaban peores notas en matemáticas, ciencia y ortografía ${ }^{(9)}$. Estudios posteriores pudieron demostrar interesantes asociaciones de los TRS con agresividad ${ }^{(10)}$, impulsividad e hiperactividad (II).

Respecto a la impulsividad e hiperactividad, se ha planteado que pudiera estar directamente relacionada con los TRS y ser -a su vez- un mecanismo planteable para el desarrollo posterior del mal rendimiento escolar. En un estudio realizado por Chervin y colaboradores en 229 niños entre 2 a 13 años, se pudo demostrar que la presencia de ronquido predecía la aparición de un trastorno de hiperactividad e impulsividad ${ }^{(2)}$. La presencia de ronquido habitual o ronquido fuerte se asociaba a un riesgo mayor en hasta 4,5 veces para el desarrollo de hiperactividad o impulsividad ${ }^{(12)}$.

Inicialmente se postuló que solamente el extremo de mayor severidad de los TRS, es decir las apneas obstructivas del sueño, provocaban tales consecuencias neurocognitivas. Sin embargo, se ha podido demostrar en numerosos estudios que el ronquido persistente, frecuente, definido como un ronquido audible por más de 3 veces a la semana, puede estar relacionado con consecuencias de similar magnitud(13-17). Se ha sugerido por lo anterior, dejar de lado el término de "ronquido benigno" y denominara estos pacientes "roncadores primarios", ya que no estarían exentos de consecuencias.

Estudios realizados recientemente por nuestro grupo en Chile pudieron demostrar -al igual que estudios extranjerosuna asociación significativa del ronquido habitual con la escala de Conners ${ }^{(18)}$ para hiperactividad $(r=0,47)$ en 523 escolares. La asociación con un mal rendimiento escolar específico en los ramos de castellano y en el promedio general se mantuvo incluso después de ajustar por factores biodemográficos asociados $^{(19)}$. El hallazgo cobra especial relevancia, ya que estas diferencias se mantuvieron en distintos grupos etarios, desde enseñanza básica a media(19). Hace algunos años, Beebe realizó un extenso metanálisis respecto a la evidencia existente sobre la asociación entre TRS y consecuencias neurocognitivas ${ }^{(20)}$. En este metanálisis se concluye que existe evidencia suficiente para demostrar un patrón temporal en el cual los TRS son una causa biológicamente plausible para el desarrollo de consecuencias neurocognitivas a largo plazo.

\section{MECANISMOS}

Los mecanismos por los cuales los TRS producen consecuencias neurocognitivas a corto y largo plazo no están del todo aclarados. Se han postulado 3 mecanismos que hipotéticamente estarían relacionados: disrupción, fragmentación del sueño e hipoxemia intermitente. La hipoxemia intermitente durante el sueño se ha demostrado que produce una pérdida neuronal en modelos animales. Recientemente, esta pérdida también se pudo demostrar en niños roncadores mediantes técnicas de neuroimagen avanzadas(21). Las zonas más afectadas son la corteza frontal e hipocampo ${ }^{(21,22)}$.

Sin embargo, probablemente la fragmentación del sueño producida por microdespertares múltiples pudiera ser la principal causa involucrada en el desarrollo de consecuencias a corto y largo plazo. La presencia de microdespertares gatillaría una cadena de eventos anormales durante el sueño, como la liberación de catecolaminas, vasoconstricción cerebral y periférica, además de la liberación de mediadores de stress oxidativo. Todos estos efectos podrían tener un efecto sumativo. La figura 2 resume los mecanismos involucrados en el desarrollo de consecuencias neurocognitivas asociadas a los TRS.

\section{TRATAMIENTO}

El tratamiento de los TRS se basa en disminuir o paliar el aumento de la resistencia de la vía aérea superior. Existen tres formas de tratamiento estudiadas en los TRS: i) tratamiento medicamentoso, ii) quirúrgico; iii) ventilación no invasiva. El tratamiento medicamentoso se basa en el uso de corticoides inhalados nasales o de montelukast. Recientemente, una revisión sistemática fue publicada respecto a la evidencia en el uso de corticoides nasales y otros agentes para esta enfermedad $^{(23)}$. El tratamiento quirúrgico (en general adenotonsi- 


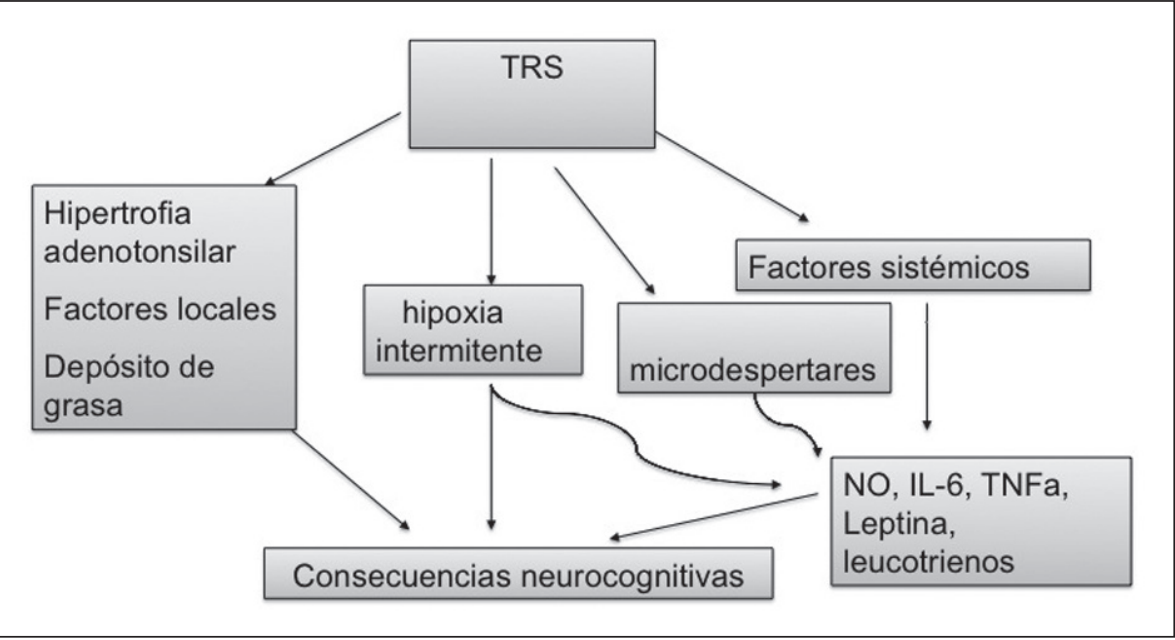

Figura 2. Mecanismos involucrados en el desarrollo de consecuencias neurocognitivas asociadas a TRS. lectomía) ha sido recomendado como primera línea en por la guías clínicas extranjeras ${ }^{(24)}$. Sin embargo, existe cada vez más evidencia que la cirugía no es infalible, en especial en grupos de alto riesgo como los pacientes obesos ${ }^{(25)}$. Un estudio multicéntrico que incluyó a 578 niños demostró un tasa de éxito post adenotonsilectomía baja, considerando eso sí una alta tasa de prevalencia de obesidad en la muestra(25). El uso de ventilación no invasiva está reservado actualmente para cuadro muy severos, obesidad o pacientes con patologías de base como por ejemplo los niños con enfermedades neuromusculares ${ }^{(26)}$. Beebe plantea en su revisión sobre los efectos neurocognitivos de los TRS, que el efecto del tratamiento sobre las consecuencias de los TRS pudiera depender de la precocidad con la cuál este sea iniciado ${ }^{(20)}$. Es decir, existe la hipótesis que un tratamiento muy tardío pudiera no lograr revertir del todo los daños causados por los TRS mediante los mecanismos anteriormente señalados.

\section{CONCLUSIONES}

Ante la evidencia de las significativas consecuencias que pudieran tener los TRS se sugiere a todos los clínicos prestar atención a todo niño que consulte por ronquido, es más, este síntoma se debiera de pesquisar activamente. Por otro lado, aquellos niños que consultan por hiperactividad, problemas escolares o de conducta debiera de preguntarse dirigidamente por síntomas que sugieran TRS. El diagnóstico y tratamiento de los TRS debieran de ser iniciado lo más precoz posible.

\section{REFERENCIAS}

I. Lumeng JC, Chervin RD. Epidemiology of pediatric obstructive sleep apnea. Proc Am Thorac Soc 2008; 5: 242-52.

2. Guilleminault C, Kim YD, Palombini L, Li K, Powell N. Upper airway resistance syndrome and its treatment. Sleep 2000; 23 Suppl 4: S197-200.

3. Iber C, Ancoli-Israel S, Chesson A, and Quan SF for the American Academy of Sleep Medicine. The AASM Manual for the Scoring of Sleep and Associated Event: Rules, Terminology and Technical Specifications. I st ed. Westchester, Illinois: American Academy of Sleep Medicine; 2007.

4. Marcus CL, Greene MG, Carroll JL. Blood pressure in children with obstructive sleep apnea. Am J Respir Crit Care Med 1998; 157: 1098-103.

5. Vgontzas AN, Papanicolaou DA, Bixler EO, Hopper K, Lotsikas A, Lin HM, et al. Sleep apnea and daytime sleepiness and fatigue: relation to visceral obesity, insulin resistance, and hypercytokinemia. J Clin Endocrinol Metab 2000; 85: I I 5 I-8.

6. Hill CM, Hogan AM, Karmiloff-Smith A. To sleep, perchance to enrich learning? Arch Dis Child 2007; 92: 637-43.

7. Ferri R, Drago V, Arico D, Bruni O, Remington RW, Stamatakis K, et al. The effects of experimental sleep fragmentation on cognitive processing. Sleep Med 2010; 11 : 378-85.

8. Gozal D. Sleep-disordered breathing and school performance in children. Pediatrics 1998; 102: 616-20.

9. Urschitz MS, Guenther A, Eggebrecht E, Wolff J, Urschitz-Duprat PM, Schlaud M, et al. Snoring, intermittent hypoxia and academic performance in primary school children. Am J Respir Crit Care Med 2003; 168: 464-8.

10. Urschitz MS, Eitner S, Guenther A, Eggebrecht E, Wolff J, UrschitzDuprat PM, et al. Habitual snoring, intermittent hypoxia, and impaired behavior in primary school children. Pediatrics 2004; I |4: |04|-8.

I I. Huang YS, Guilleminault C, Li HY, Yang CM, Wu YY, Chen NH. Attention-deficit/hyperactivity disorder with obstructive sleep apnea: a treatment outcome study. Sleep Med 2007; 8: 18-30.

12. Chervin RD, Ruzicka DL, Archbold KH, Dillon JE. Snoring predicts hyperactivity four years later. Sleep 2005; 28: 885-90.

13. Blunden S, Lushington K, Lorenzen B, Martin J, Kennedy D. Neuropsychological and psychosocial function in children with a history of snoring or behavioral sleep problems. J Pediatr 2005; 146: 780-6.

14. Kennedy JD, Blunden S, Hirte C, Parsons DW, Martin AJ, Crowe $E$, et al. Reduced neurocognition in children who snore. Pediatr Pulmonol 2004; 37: 330-7.

15. O'Brien LM, Mervis CB, Holbrook CR, Bruner JL, Klaus CJ, Rutherford J, et al. Neurobehavioral implications of habitual snoring in children. Pediatrics 2004; I 14: 44-9. 
16. Brockmann PE, Urschitz MS, Schlaud M, Poets CF. Primary snoring in school children: prevalence and neurocognitive impairments. Sleep \& breathing = Schlaf \& Atmung 2012; 16: 23-9.

17. Ali NJ, Pitson D, Stradling JR. Sleep disordered breathing: effects of adenotonsillectomy on behaviour and psychological functioning. Eur J Pediatr 1996; 155: 56-62.

18. Conners CK, Sitarenios G, Parker JD, Epstein JN. The revised Conners' Parent Rating Scale (CPRS-R): factor structure, reliability, and criterion validity. J Abnorm Child Psychol 1998; 26: 257-68.

19. Brockmann PE, Bertrand P, Pardo T, Cerda J, Reyes B, Holmgren NL. Prevalence of habitual snoring and associated neurocognitive consequences among Chilean school aged children. Int J Pediatr Otorhinolaryngol 2012; 76: 1327-31.

20. Beebe DW. Neurobehavioral morbidity associated with disordered breathing during sleep in children: a comprehensive review. Sleep 2006; 29: III5-34.

21. Halbower AC, Degaonkar M, Barker PB, Earley CJ, Marcus CL, Smith $\mathrm{PL}$, et al. Childhood obstructive sleep apnea associates with neuropsychological deficits and neuronal brain injury. PLoS Med 2006; 3: 1391-402.
22. Halbower AC, Ishman SL, McGinley BM. Childhood obstructive sleep-disordered breathing: a clinical update and discussion of technological innovations and challenges. CHEST 2007; 132: 2030-4I.

23. Kuhle S, Urschitz MS, Eitner S, Poets CF. Interventions for obstructive sleep apnea in children: A systematic review. Sleep Med Rev 2009; | 3: |23-31.

24. American Academy of Pediatrics, Section on Pediatric Pulmonology, Subcommittee on Obstructive Sleep Apnea Syndrome. Clinical practice guideline: diagnosis and management of childhood obstructive sleep apnea syndrome. Pediatrics 2002; 109: 704-12.

25. Bhattacharjee R, Kheirandish-Gozal L, Spruyt K, Mitchell RB, Promchiarak J, Simakajornboon $\mathrm{N}$, et al. Adenotonsillectomy outcomes in treatment of obstructive sleep apnea in children: a multicenter retrospective study. Am J Respir Crit Care Med 20 I0; 182: 676-83.

26. Brockmann P, Salinas P, Holmgren N, Prado F. Utilidad de la clínica para el diagnóstico de trastornos respiratorios del sueño en niños con enfermedades neuromusculares. Rev Chil Pediatr 2011; 4: 319-28. 